\title{
II Methode
}

\section{Untersuchungskontext und Studiendesign}

Für die vorliegende Arbeit wird auf die Daten von zwei Erhebungswellen des längsschnittlichen Teilprojektes A2 „Die Entstehung und Entwicklung devianten und delinquenten Verhaltens im Lebensverlauf und ihre Bedeutung für soziale Ungleichheitsprozesse" im Rahmen des durch die DFG geförderten SFB 882 „Von Heterogenitäten zu Ungleichheiten“ zurückgegriffen.

Das Teilprojekt ist eine Zusammenarbeit der Fakultät für Soziologie der Universität Bielefeld unter der Leitung von Prof. Dr. Jost Reinecke und des Psychologischen Instituts der FriedrichAlexander-Universität Erlangen-Nürnberg unter der Leitung von Prof. Dr. Mark Stemmler. Dieses Projekt zielt darauf ab, die Entstehung und Verfestigung devianter und delinquenter Verhaltensmuster im Lebensverlauf unter zeitgleicher Berücksichtigung soziologischer und psychologischer Perspektiven zu untersuchen.

Methodisch wurde die Studie durch ein Kohorten-Sequenz-Design im Längsschnitt mit jeweils zwei Kohorten und an zwei Standorten durchgeführt. Dank diesem Design ist ab der ersten Untersuchungswelle der querschnittliche Vergleich der jüngeren und älteren Befragten ermöglicht. Die jährliche Befragung mehrerer Kohorten schafft zudem zahlreiche längsschnittliche Auswertungsstrategien. Befragt wurden Schüler der Haupt-, Real- und Gesamtschulen und Gymnasien in Dortmund sowie Schüler der Mittelschulen in Nürnberg, die vergleichbar mit den Dortmunder Hauptschulen sind. Die Ausgangsstichprobe dieses Teilprojektes sind die Fünft- sowie Neuntklässler aus dem Jahr 2012. Allerdings gestatten die für die vorliegende Arbeit benötigten Variablen eine Untersuchung erst ab der zweiten Untersuchungswelle dieses Teilprojektes, $d$. h. die zweite Untersuchungswelle des Teilprojektes gilt als erste Untersuchungswelle für das Dissertationsprojekt. Nachfolgend wird mit der ,ersten Untersuchungswelle“ für die vorliegende Arbeit jene gemeint, falls keine weitere Erklärung vorhanden ist. 